\title{
Usability-Testing in agilen Entwicklungsprojekten
}

\author{
Alexander Rösler \\ usability.de \\ Plaza de Rosalia 4 \\ 30449 Hannover \\ alexander.roesler@usability.de
}

\author{
Michaela Thölke \\ usability.de \\ Plaza de Rosalia 4 \\ 30449 Hannover \\ michaela.thoelke@usability.de
}

\begin{abstract}
Agile Softwareentwicklung ist heute in vielen Unternehmen angekommen. Statt lange zu planen und zu spezifizieren, sollen Anforderungen innerhalb kürzester Zeit in funktionierender Software aufgehen. Dies stellt Usability Dienstleister vor neue Herausforderungen. Die Durchführung eines klassischen Usability-Tests mit Erstellung eines Prototyps, Vorbereitung, Durchführung und schriftlicher Dokumentation dauert zu lang und wird den Anforderungen agiler Softwareentwicklung nicht gerecht. In einem Erfahrungsbericht aus zwei Projekten für den Energiekonzern RWE und das Expat Netzwerk InterNations berichten wir, wie wir durch ein neues schlankes Testing-Verfahren auch in agilen Projekten schnelles und gleichzeitig wertvolles Usability-Feedback liefern können.
\end{abstract}

\section{Keywords}

Usability-Test, Agile Entwicklung

\section{Einleitung}

Agile Softwareentwicklung ist heute in vielen Unternehmen angekommen. Statt lange zu planen und $\mathrm{zu}$ spezifizieren, sollen Anforderungen innerhalb kürzester Zeit in funktionierender Software aufgehen. Dies stellt Usability Dienstleister und In-House Usability Experten vor neue Herausforderungen. Der Projektplan sieht im Regelfall keine zweiwöchige Entwicklungspause für die Durchführung eines klassischen Usability-Tests mit Erstellung eines Prototyps, Vorbereitung, Durchführung und schriftlicher Dokumentation vor. Nach dem Motto fail fast, learn fast soll im Optimalfall innerhalb von 1-2 Tagen Usability-Feedback zum aktuellen Entwicklungsstand eingeholt werden, um direkt Optimierungen vornehmen zu können. 
Um auf diese neuen Anforderungen zu reagieren, haben wir bei usability.de ein neues schlankes Testing-Verfahren entwickelt. Im Kern setzt dieses Modell auf eine enge Zusammenarbeit mit den Stakeholdern auf Kundenseite. Statt als Dienstleister fertige Ergebnisse in Form eines ausführlichen Reports und eines Highlightvideos zu liefern, wird der Kunde direkt bei der Vorbereitung, Beobachtung und Auswertung mit einbezogen.

\section{Best Practices}

Dies klingt im Prinzip erstmal ganz einfach und logisch. In der Praxis haben wir jedoch gelernt, dass es bei der Arbeitsteilung und Herangehensweise einige Dinge zu beachten gilt. In einem praktischen Erfahrungsbericht möchten wir unsere Learnings deshalb gerne teilen.

\section{Wer übernimmt die Vorbereitung?}

In nicht agilen Testing Projekten achten wir darauf, dass die Vorbereitung und das Entwickeln von Szenarien immer auf unserer Seite liegen. Mit dem Kunden besprechen wir, welche Fragestellungen durch den Test beantwortet werden sollen und welche Hauptanwendungsfälle die Nutzer normalerweise mit der Anwendung verfolgen. Das eigentliche Ausformulieren nicht suggestiver, gut verständlicher Szenarien erfolgt dann aber durch uns als Usability-Experten.

In agilen Testing Projekten funktioniert dies leider so nicht immer. Teilweise ist bis kurz vor dem Test noch unklar, welche Anwendungsbestandteile überhaupt funktionsfähig sein werden. Manchmal ändert sich sogar der Scope des Tests noch kurzfristig, weil sich neue Feature Wünsche ergeben. Um Kommunikationsaufwände gering $\mathrm{zu}$ halten und sicherzugehen, dass die Aufgaben später funktionieren, erarbeiten deshalb unsere Kunden Vorschläge für die Aufgabenszenarien.

Achtung Falle! Als Usability-Experten wissen wir, wie schwierig es sein kann gute Szenarien zu entwickeln. Die Szenarien sollen leicht verständlich formuliert sein, den Nutzer zur Interaktion mit der Anwendung auffordern und trotzdem möglichst wenig von der Aufgabenlösung verraten. Ohne Coaching und Kontrolle geht es deshalb nicht. Wir briefen unsere Kunden deshalb detailliert, was es beim Schreiben von Szenarien zu beachten gilt. Grundsätzlich planen wir außerdem mindestens eine Feedbackschleife schon vor dem PreTest ein, um sicherzugehen, dass die Qualität der Szenarien stimmt.

Die finale Qualitätskontrolle und Abnahme der Testszenarien liegt dann natürlich bei uns! 


\section{Wie geht man damit um, dass der Testgegenstand erst kurz vor dem Test fertiggestellt wird?}

Agile Softwareentwicklung ist im Optimalfall schnell. Statt monatelang an einem Konzept zu schreiben, um dann in das Design und die Entwicklung einzusteigen, entwickeln unsere Kunden innerhalb weniger Wochen Teile komplexer Anwendungen. Dies hat zur Folge, dass wir als Agentur oft erst wenige Tage bis Stunden vor der Testdurchführung den finalen Testgegenstand zur Verfügung gestellt bekommen.

Achtung Falle! In der Vergangenheit führte das späte Liefern des Testgegenstands immer wieder zu Problemen.

In einem Fall konnten wir die wesentliche Fragestellung des Kunden nicht beantworten, weil der Testgegenstand nicht ausreichend interaktiv war. So war es unter anderem ein Ziel, die Seitennavigation der Website $\mathrm{zu}$ testen, der Nutzer konnte jedoch nicht zwischen den verschiedenen Seitenteilen navigieren.

In einem Fall wurde das Testergebnis durch den gelieferten Testgegenstand verzerrt. Ein Kalender Widget sollte getestet werden - das im Kalender $\mathrm{zu}$ findende Element wurde jedoch direkt beim Öffnen des Kalenders angezeigt (so dass natürlich jeder Teilnehmer „erfolgreich“war).

Um diese Probleme zu umgehen, empfehlen wir, die Szenarien für den Test bereits vor der Finalisierung des Testgegenstands (und häufig schon vor der Entwicklung) festzulegen. Durch die vorgegebenen Szenarien wird sehr klar was genau an Interaktivität für den Test vorhanden sein muss. Außerdem ist es uns wichtig, bereits früh in der Entwicklung mit einbezogen werden, so dass wir auf solche Probleme frühzeitig hinweisen können.

\section{Wen genau lädt man zur Testdurchführung ein?}

Ganz einfach: Alle Personen, die an der Entwicklung beteiligt sind.

Ziel ist es, das am nächsten Tag sofort mit den an der Software notwendigen Anpassungen begonnen werden kann. Dies ist nur möglich, wenn alle, die Anpassungen vornehmen sollen, informiert sind. Die Information erfolgt über die Teilnahme an den Tests (alle haben das Gleiche gesehen). Das Reporting beschränkt sich auf das Nötigste und dient dabei als Erinnerungsstütze.

Das ist das Ziel. Die Realität sieht meist so aus, dass ein bis zwei feste Mitarbeiter aus dem Kundenprojektteam dabei sind und weitere Mitarbeiter nur einzelne Testsessions sehen (remote oder live). Bei der abschließenden Besprechung sollten dann aber möglichst alle dabei sein.

Achtung Falle! Der Kunde denkt, es sei nicht notwendig an ALLEN Tests teilzunehmen. Besonders mit den wenigen Teilnehmern (wir beschränken uns oft auf einen Testtag) je Test in einem agilen Setting ist es jedoch wichtig, alle Tests zu sehen, um ein klares Gesamtbild zu erhalten. Sonst bleibt nur die Auswahl der gesehenen Tests im Gedächtnis, was zu einer 
verzerrten Wahrnehmung führen kann. Daher sollten ein bis zwei Mitarbeiter des Kunden fest dabei sein, damit ein klares Gesamtbild in das Unternehmen weitergetragen wird.

Achtung Falle! Der Kunde denkt, die umzusetzende Agentur müsse nicht an den Test teilnehmen. Das kann klappen, kann aber dazu führen, dass die Agentur wichtige Findings nicht versteht oder nicht ernst genug nimmt. Da das Reporting sehr knapp ist, ist mehr Kommunikationsaufwand vom Kunden zu Agentur notwendig.

\section{Wie genau bereitet man Kunden auf die Beobachtung der Testsessions vor?}

Generell empfehlen wir all unseren Kunden, bei Usability-Tests zuzuschauen. Der Kunde erlebt so einerseits aus erster Hand, welche Schwierigkeiten Nutzer bei der Lösung der Testaufgaben haben, andererseits ermöglicht uns die Anwesenheit des Kunden, mehr über den bisherigen Verlauf des Projekts und bisher getroffene Entscheidungen zu erfahren. Bei normalen Usability-Tests weisen wir unsere Kunden darauf hin, mehr auf das Verhalten zu achten als auf das gesprochene Wort und nicht nach der ersten Testperson bereits alle Schlüsse zu ziehen.

Bei iterativen, agilen Tests genügt es nicht, wie bei normalen Tests, den Kunden auf die Beobachtung während der Tests hinzuweisen, sondern hier soll der Kunde aktiv mitarbeiten. Was heißt das?

Da wir gemeinsam die beobachteten Probleme sammeln und beurteilen wollen, bitten wir jeden Projektbeteiligten während der Tests alle beobachteten Probleme schriftlich festzuhalten.

Achtung Falle! Viele Beobachter neigen dazu, wirklich jede einzelne Bemerkung der Teilnehmer aufzuschreiben. Das führte zu Beginn oft zu langwierigen Nachbesprechungen der einzelnen Tests. Kunden fällt es oft schwer zwischen echten Usability-Problemen, generellem Feedback oder Wünschen für derzeit nicht vorhandene Features zu unterscheiden. Wesentliche Beobachtungen gehen dadurch vielleicht unter. Hier sollte man für die ersten Tests deutlich mehr als eine halbe Stunde für die Besprechung einplanen. Des Weiteren erklären wir schon vor der Durchführung des ersten Tests wie genau ein UsabilityProblem definiert ist.

Achtung Falle! Anfangs haben wir die Kunden gebeten auf Post Its ihre Beobachtungen zu notieren (ein Problem je Post It). Inzwischen sind wir davon abgerückt, da die Masse an Post Its besprochen werden will. Zudem kann man ein Post It nicht einfach aussortieren (oder es erfordert einiges an Fingerspitzengefühl). Dazu später mehr! 


\section{Wie nimmt man Kunden bei der Auswertung der Testsessions vernünftig mit?}

Nach jedem Test haben wir eine Pause von mindestens 30 Minuten. Diese Pause dient normalerweise bei uns dazu, das Testsetting wiederherzustellen (z.B. Browser Cache leeren, andere Vorbereitungen zu absolvieren), einen Puffer zu haben (falls ein Teilnehmer zu spät oder zu früh kommt) und sich mit dem Kunden auszutauschen.

Bei iterativen, agilen Tests besprechen wir in der Pause zusätzlich die Erkenntnisse aus dem letzten Test. Dazu versammelt sich das gesamte Projektteam im Beobachtungsraum, in den die Test Sessions per Livestream übertragen werden, zur Nachbesprechung. Mit Hilfe des Testleitfadens leitet der Moderator die Auswertung an. Chronologisch wird der Test von Anfang bis Ende besprochen und alle Teilnehmer dürfen ihre Beobachtungen teilen.

Wenn ein Problem beobachtet wurde und sich die Runde einig ist, wird dieses auf einem Post-it festgehalten und an unsere Auswertungswand gehängt. Hilfreich ist es dabei, Probleme gleich nach Seitenbereichen zu clustern.

Achtung! Falle Wie bereits im Abschnitt „Wie genau bereitet man die Kunden auf die Beobachtung der Testsettings vor" beschrieben, erklären wir unseren Kunden anhand von Beispielen bereits vor den Tests, was Probleme, Wünsche, Beobachtungen und Lösungen sind. Wir hatten anfangs sehr mit der Fülle an beobachteten „Problemen“ zu kämpfen. Die Gruppe spielt sich aber sehr schnell ein. Um dies zu unterstützen, ist es sinnvoll aufeinanderfolgende Projekte mit den gleichen Consultants zu besetzen.

Jedes Problem wird bei uns außerdem mit einem Schweregrad bewertet. Wie schwerwiegend war das Problem für den Nutzer? Hierfür haben wir Definitionen entwickelt, die vor der Auswertung erklärt werden. Diese Definitionen beschreiben eindeutig, wann genau ein Problem als kritisch und wann nur als geringfügig einzustufen ist.

Achtung Falle: Analog zur Sammlung der Probleme haben wir zu Beginn auch die Schweregradbewertung gemeinsam mit dem Kunden vorgenommen („Was denkt ihr, wie bewertet ihr das Problem?“). Dies hat den Vorteil, dass der Kunde selbst darüber nachdenkt und auch mit der Bewertung konform geht. Andererseits hängen an der Beurteilung häufig auch andere projektrelevante Entscheidungen, wie z.B. ob ein Bereich ein erneutes Mal überarbeitet wird oder nicht. Insbesondere wenn die umsetzende Agentur ebenfalls anwesend ist, kann dies zu hitzigen Diskussionen führen. Eine objektive Beurteilung von Problemen fällt dann schwer. Deshalb sind wir inzwischen dazu übergegangen, dass wir als Experten die finale Entscheidung treffen, aber die Meinungen und Einschätzungen gern hören. Den Goldstandard bilden dabei unsere Schweregraddefinitionen. 


\section{Wie genau werden die Testergebnisse und Lösungsvorschläge dokumentiert, um später schnell verwertbar zu sein?}

Bei normalen Tests erhält der Kunde bei uns ein ausführliches Reporting mit Problembeschreibung, Empfehlung zur Lösung der Probleme (Best Practice oder Wireframes), Screenshots, Highlightvideo usw. Das Erstellen dieses Ergebnisberichts dauert im Normalfall mehrere Tage.

Ziel der agilen Tests ist es, dass der Kunde sofort weiter entwickeln kann. Alles Wissen dafür hat er durch die Beobachtung und Beteiligung an der Auswertung, trotzdem ist eine kleine Form der Dokumentation hilfreich, um sicherzustellen, dass auch alle Punkte beachtet und bearbeitet werden können.

Wir führen dazu immer ein Debriefing durch: Nach dem letzten Test reservieren wir ein bis drei Stunden, um noch einmal die Probleme zusammenzufassen und dann auch das erste Mal über Lösungen zu sprechen. Dabei wird außerdem kurz mitprotokolliert: Problembeschreibung, Schweregrad, diskutierte Lösungen, evtl. eine Priorität. Außerdem fertigen wir Fotos der Post Its an.

Achtung Falle! Oben ist natürlich der Idealfall beschrieben. In einem Konzern möchten natürlich auch andere Stakeholder wissen, wie der Test lief und dafür eignet sich o.g. Kurzdokumentation nur bedingt. Hier muss man in Absprache mit dem Kunden ggf. doch wieder auf das übliche Reporting zurückgreifen. Trotzdem sehen wir hier den Vorteil, dass die weitere Entwicklung dadurch nicht behindert wird (und der Report zur Formsache wird).

Ähnliches gilt übrigens für das Highlightvideo: Wenn doch nicht alle Mitarbeiter zuschauen können, dann kann es hilfreich sein, wenigstens Eindrücke über die wichtigsten Erkenntnisse zu vermitteln. Statt eines langen Reports haben wir sehr gute Erfahrungen mit der Erstellung eines kurzen Highlightvideos gemacht. So kann man weitere Stakeholder auf Kundenseite schnell ins Thema holen.

\section{Verlieren die Ergebnisse durch die Schnelligkeit des Verfahrens an Qualität?}

Uns ist es in jedem Projekt wichtig, den Kunden belastbare, nachvollziehbare und qualitativ hochwertige Ergebnisse zu liefern und wir haben uns natürlich auch gefragt, ob wir durch die Schnelligkeit des Verfahrens nicht an Qualität verlieren. Immerhin erhält der Kunde innerhalb von 1-2 Tagen die Ergebnisse seines Usability-Tests und nicht wie sonst üblich nach ca. einer Woche.

Woran sparen wir und wie stellen wir sicher, dass dies keinen negativen Einfluss auf die Qualität hat? 
Vorbereitungszeit: Der Kunde übernimmt einen Teil der Vorbereitung, aber wir führen eine Qualitätskontrolle durch und beraten bei der Erstellung der Szenarien. Das letzte Wort bei der Formulierung der Szenarien haben wir.

Teilnehmerzahl: Statt den üblichen 8 Teilnehmern testen wir mit 5 Teilnehmern und können so auf einen Durchführungstag reduzieren. Durch iteratives Testen stellen wir sicher, dass wichtige Bereiche immer wieder überprüft werden (z.B. Startseite).

Auswertungszeit: Statt die Auswertung nach den Tests durchzuführen, besprechen wir die Ergebnisse mit den Kunden gemeinsam zwischen den Tests. Durch die Beteiligung von zwei erfahrenen Usability Consultants stellen wir sicher, dass Ergebnisse korrekt interpretiert werden.

Dokumentation: Die Hauptersparnis resultiert aus der reduzierten Dokumentation. Statt ausführlichem Reporting kommt hier lediglich ein kurzes Word-Dokument zum Einsatz. Da der Kunde jedoch alle Tests und Besprechungen erlebt hat und die Ergebnisse sofort in eine Umsetzung fließen, wird lediglich eine Erinnerungsstütze benötigt und keine Dokumentation, die auch für Unbeteiligte nachvollziehbar ist.

\section{Fazit}

"Working software over comprehensive documentation."

(Manifesto for Agile Software Development)

Das Testsetting muss an die agile Herangehensweise angepasst werden. Vor allem die Beteiligung des Kunden am Prozess erfordert einige Vorbereitungen. Wenn die o.g. Fallen jedoch beachtet werden, stehen agile Testingprojekte in nichts den normal üblichen Usability-Tests nach.

Agiles Testing eignet sich hervorragend in agilen Entwicklungsprojekten, wo nicht lange auf ausführliche Reports gewartet werden kann. Das Ziel ist in kürzester Zeit verwertbares Nutzerfeedback zu erhalten.

Aus unserer Einschätzung heraus, ist die Qualität der Testergebnisse mit normalen Tests absolut vergleichbar. Sie hat sogar den Vorteil, dass der Kunde die Testergebnisse viel besser versteht, da er direkt bei der Ermittlung der Ergebnisse beteiligt war.

Wichtig ist, den Kunden deutlich zu machen, wo die Zeiteinsparung herkommt: Wir sparen Dokumentationszeit, die der Kunde durch Anwesenheitszeiten und Mitarbeit kompensieren muss. Sprich: Wir verteilen die Aufwände nur anders. Unterm Strich bleibt der Aufwand der gleiche oder ist sogar höher (weil mehr Personen involviert sind). 